\section{The translational potential of virtual reality}

dissociation,
particularly
depersonal-
ization and
derealiza-
tion, might
be important
facilitators
for suicide
behaviour

Feelings of depersonalization (feeling detached from the self and the body) and derealization (feeling that the external world is unreal or altered) predict the decision to engage in suicide in a virtual reality scenario according to new research published in Journal of Affective Disorders. These findings suggest that certain facets of dissociation are important facilitators for suicide.

Suicide is a leading cause of death worldwide, but the risk factors that correlate with suicidal ideation are only weak predictors of suicidal behaviour. Thus, little is known about the transition between ideation and a suicide attempt. Moreover, owing to ethical and feasibility considerations, suicide research relies heavily on retrospective self-report, which limits understanding of causality. "I wanted to do this project because I am passionate about trying to find novel ways to examine suicide risk and augment self-report measures", says graduate student and study lead author Nicole Caulfield.

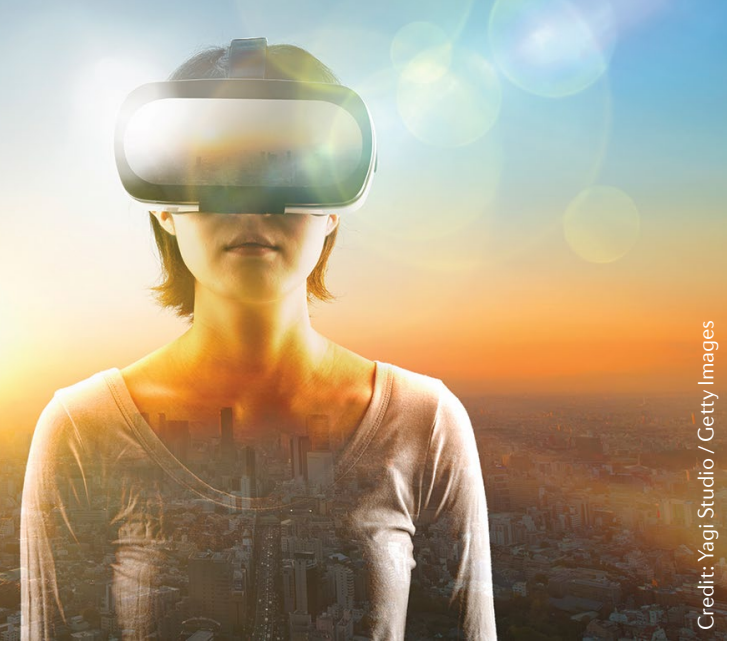

In the study, undergraduate participants wore a virtual reality headset and virtually experienced entering an elevator, going up a few hundred feet and watching as the doors opened to a plank extending over open space between skyscrapers. They were then given the choice whether or not to walk out onto the plank and step off it. They were told that if they stepped off the plank, they were choosing to die by suicide. If participants chose to step off the plank, they virtually fell to the ground below and the screen faded to white. Participants stood on an actual plank during this task to make the experience immersive.

For half the participants, feelings of dissociation were induced using virtual reality prior to engaging in the scenario described above. To induce dissociation, participants were instructed to spin in a circle or move their head in different directions while visual feedback was augmented, such that participants viewed themselves moving in the third person or moving in an opposite direction to their actual movement. Thus, the researchers leveraged features of virtual reality to simulate both the experience of a suicide opportunity and their key facilitator of interest, dissociation.

"Dissociation can be a potential facilitator [of suicide] via increased insensitivity to pain and intensified disconnect from the body, allowing someone to feel more capable of harming themselves", explains Caulfield. Indeed, the main finding of the study is that acute feelings of depersonalization and derealization were associated with choosing virtual suicide. This association remained significant after controlling for impulsivity (which might reflect an overall desire to experience all scenario options) and simulation sickness (which might influence wanting to complete the final step of the simulation).

Ethical considerations must be considered when studying suicide. Importantly, participants in this study were screened for immediate suicide risk and current or past psychotic disorders prior to participation. Moreover, as Caulfield points out, the results of this study showed that, on average across all participants, suicide risk decreased after engaging in the virtual suicide scenario. This is consistent with other studies suggesting that exposure to suicide-related content in research contexts is consistently associated with small reductions in suicide risk.

Although behaviours during a virtual reality simulation differ in important ways from engaging in actual suicide behaviour, "using virtual reality for suicide research holds a lot of promise for future multi-method examinations of suicide risk", says Caulfield. In addition to showing that dissociation, particularly depersonalization and derealization, might be important facilitators for suicide behaviour, "this investigation adds to growing evidence that virtual reality is an ethical method that can recreate certain sensations and situations that one might experience when engaging in a suicide attempt."

Jenn Richler

ORIGINAL ARTICLE Caulfield, N. M. et al. Exploring dissociation as a facilitator of suicide risk: a translational investigation using virtual reality. J. Affect. Disord. 297, 517-524 (2022) RELATED ARTICLE Blades, C. A. et al. The benefits and risks of asking research participants about suicide: a meta-analysis of the impact of exposure to suicide content. Clin. Psychol. Rev. 64 , 1-12 (2018) 